\title{
LOCAL PRESSURE CORRECTION FOR THE STOKES SYSTEM*
}

\author{
Malte Braack and Utku Kaya \\ Mathematical Seminar, University of Kiel, Germany \\ Email:braack@math.uni-kiel.de, kaya@math.uni-kiel.de
}

\begin{abstract}
Pressure correction methods constitute the most widely used solvers for the timedependent Navier-Stokes equations. There are several different pressure correction methods, where each time step usually consists in a predictor step for a non-divergence-free velocity, followed by a Poisson problem for the pressure (or pressure update), and a final velocity correction to obtain a divergence-free vector field. In some situations, the equations for the velocities are solved explicitly, so that the numerical most expensive step is the elliptic pressure problem. We here propose to solve this Poisson problem by a domain decomposition method which does not need any communication between the sub-regions. Hence, this system is perfectly adapted for parallel computation. We show under certain assumptions that this new scheme has the same order of convergence as the original pressure correction scheme (with global projection). Numerical examples for the Stokes system show the effectivity of this new pressure correction method. The convergence order $\mathcal{O}\left(k^{2}\right)$ for resulting velocity fields can be observed in the norm $l^{2}\left(0, T ; L^{2}(\Omega)\right)$.
\end{abstract}

Mathematics subject classification: 76D07, 65M55.

Key words: Stokes system, Navier-Stokes, Pressure correction, Finite elements.

\section{Introduction}

Pressure correction methods are the most widely used methods to solve the time-dependent Navier-Stokes equations, because they open the possibility to decouple the momentum equation from the divergence equation and, hence, lead to the possibility to use different solution techniques for obtaining the velocity and the pressure.

Since the pioneering work of Chorin and Temam $[2,8]$ many different pressure correction methods have been proposed. An extensive overview was provided by Guermond et al [3]. These methods commonly contain a prediction step for a not necessarily divergence-free velocity field, followed by a Poisson problem for the pressure (or a pressure update), and finally a projection of the previously computed velocity onto a divergence free one. In certain applications with the need of small time steps due to accuracy reasons, the predictor step can be formulated in an explicit way. Of course this requires an explicit treatment of the convective and diffusive term, so that severe time step restrictions are needed for stability. However, this is acceptable for typical applications as for instance in climate research. In this case, a numerically very expensive part of the splitting scheme is the Poisson problem for the pressure (update). It is a global problem with an associated matrix to be inverted with condition number dependent on the mesh resolution. For finer meshes, the condition number becomes larger, so that many iterative solvers (such as conjugate gradient methods, Jacobi iteration and Gauss-Seidel methods) suffer in terms of convergence rates. Parallelization on multi-core computers or parallel computers

\footnotetext{
* Received September 29, 2018 / Revised version received February 15, 2019 / Accepted June 6, 2019 /

Published online December 4, 2019 /
} 
may help to reduce the simulation time but always require suitable further iteration techniques to account for the elliptic character of the Poisson problem.

Therefore, we propose in this work an alternative splitting method which replaces the global Poisson problem for the pressure update by a number of smaller non-overlapping Poisson problems that are completely decoupled. This gives the possibility to solve the pressure correction step in parallel without any communication within the iterative linear solver. In terms of accuracy, the resulting scheme is comparable to the original scheme. To a certain extent, the new method presented and analyzed here can be considered as an extension of the coarse grid projection method introduced by Lentine et al. [7] and recently studied by Kashefi and Staples [6].

We present this modified scheme, demonstrate several properties, and show first numerical results. The starting splitting scheme is not restricted to a special one. However, for ease of presentation, we consider in this work the pressure correction scheme of Timmermans et al. [9], which has been analyzed by Shen and Guermond [4] and is considered to be among the most accurate ones.

We start with a standard and well-analyzed pressure correction method in Section 2. The new local pressure correction scheme is introduced in Section 3, where we still give freedom of a concrete projection. The a priori analysis is topic of Section 4. To this end we formulate a necessary condition of the projection (Assumption 4.1). One possible realization of the local projection is given in Section 5. We also verify the assumption needed previously to prove the error estimate. First numerical results are subject of Section 6 . We end with a short conclusion and outlook.

\section{Pressure Correction for the Stokes System}

We consider the time-dependent Stokes equations in a Lipschitz domain $\Omega \subset \mathbb{R}^{d}, d \in\{2,3\}$, homogeneous Dirichlet conditions on $\partial \Omega$ and initial velocity field $\boldsymbol{u}_{0}$. With the velocity field $\boldsymbol{u}: \Omega \rightarrow \mathbb{R}^{d}$, the pressure $p: \Omega \rightarrow \mathbb{R}$, and a forcing term $\mathbf{f}: \Omega \rightarrow \mathbb{R}^{d}$ the Stokes system reads

$$
\begin{aligned}
\partial_{t} \boldsymbol{u}-\nu \Delta \boldsymbol{u}+\nabla p & =\mathbf{f} & & \text { in } \Omega_{T}:=\Omega \times(0, T], \\
\operatorname{div} \boldsymbol{u} & =0 & & \text { in } \Omega_{T}, \\
\boldsymbol{u} & =\mathbf{0} & & \text { on } \partial \Omega \times(0, T], \\
\boldsymbol{u}(0) & =\boldsymbol{u}_{0} & & \text { in } \Omega .
\end{aligned}
$$

Here, $\nu>0$ is a positive, constant viscosity. We take $M+1$ discrete time points $0=t_{0}, t_{1}, \ldots, t_{M}$ with for simplicity a constant time step $k=t_{m}-t_{m-1}>0$ for all $m$, hence $t_{m}:=m k$ for $0 \leq m \leq M=T / k$. The outer normal on the boundary $\partial \Omega$ is denoted by $\boldsymbol{n}$. The so-called rotational incremental pressure-correction proposed by Timmermans et al. [9] starts with a given $\boldsymbol{u}_{0}$ and $p_{0}$ and reads for $m \geq 1$ :

Step 1: The velocity predictor $\tilde{\boldsymbol{u}}_{m}$ is obtained by solving the momentum equation with a $\mathrm{BDF}(2)$-scheme and a given pressure $p_{m-1}$ :

$$
\begin{aligned}
\frac{1}{2 k}\left(3 \tilde{\boldsymbol{u}}_{m}-4 \boldsymbol{u}_{m-1}+\boldsymbol{u}_{m-2}\right)-\nu \Delta \tilde{\boldsymbol{u}}_{m} & =\mathbf{f}\left(t_{m}\right)-\nabla p_{m-1} & & \text { in } \Omega \\
\tilde{\boldsymbol{u}}_{m} & =\mathbf{0} & & \text { on } \partial \Omega .
\end{aligned}
$$


Step 2: Poisson equation for the pressure correction:

$$
\begin{aligned}
-\Delta q_{m} & =-\frac{3}{2 k} \operatorname{div} \tilde{\boldsymbol{u}}_{m} & & \text { in } \Omega, \\
\nabla q_{m} \cdot \boldsymbol{n} & =0 & & \text { on } \partial \Omega, \\
\int_{\Omega} q_{m} d x & =0 . & &
\end{aligned}
$$

Step 3: Pressure update:

$$
p_{m}=p_{m-1}+q_{m}-\nu \operatorname{div} \tilde{\boldsymbol{u}}_{m} .
$$

Step 4: Velocity correction:

$$
\boldsymbol{u}_{m}=\tilde{\boldsymbol{u}}_{m}-\frac{2 k}{3} \nabla q_{m}
$$

Step 5: If $m=M$ stop. Otherwise, increase $m$ by one, increase $t$ by $k$, and go to Step 1 .

The basic features of the arising quantities are as follows: The velocity predictor $\tilde{\boldsymbol{u}}_{m}$ satisfies the (homogeneous) Dirichlet conditions on the boundary but it is in general not divergence free. In contrast to this, the velocity $\boldsymbol{u}_{m}$ is divergence free but it satisfies the boundary conditions only partially: The normal component vanishes $\boldsymbol{u}_{m} \cdot \boldsymbol{n}=0$. However, the tangential part of the velocity $\boldsymbol{u}_{m} \cdot \boldsymbol{t}$ becomes

$$
\boldsymbol{u}_{m} \cdot \boldsymbol{t}=\tilde{\boldsymbol{u}}_{m} \cdot \boldsymbol{t}-\frac{2 k}{3} \nabla q_{m} \cdot \boldsymbol{t} .
$$

Hence, it does in general not vanish. Because of $\Delta \boldsymbol{u}=-\nabla \times \nabla \times \boldsymbol{u}+\nabla \operatorname{div} \boldsymbol{u}$ and $\nabla \times \nabla \times \boldsymbol{u}_{m}=$ $\nabla \times \nabla \times \tilde{\boldsymbol{u}}_{m}$, the predictor can be eliminated out of the system. The resulting equation is a coupled system for $\boldsymbol{u}_{m}$ and $p_{m}$ :

$$
\begin{aligned}
\frac{1}{2 k}\left(3 \boldsymbol{u}_{m}-4 \boldsymbol{u}_{m-1}+\boldsymbol{u}_{m-2}\right)+\nu \nabla \times \nabla \times \boldsymbol{u}_{m}+\nabla p_{m} & =\mathbf{f}\left(t_{m}\right) & & \text { in } \Omega_{T}, \\
\operatorname{div} \boldsymbol{u}_{m} & =0 & & \text { in } \Omega_{T}, \\
\boldsymbol{u}_{m} \cdot \boldsymbol{n} & =0 & & \text { on } \partial \Omega \times(0, T] .
\end{aligned}
$$

The divergence-free velocity $\boldsymbol{u}_{m}$ can be eliminated out of the system as well, so that the system can be expressed in terms of $\tilde{\boldsymbol{u}}_{m}$ :

$$
\frac{1}{2 k}\left(3 \tilde{\boldsymbol{u}}_{m}-4 \tilde{\boldsymbol{u}}_{m-1}+\tilde{\boldsymbol{u}}_{m-2}\right)-\nu \Delta \tilde{\boldsymbol{u}}_{m}=\mathbf{f}\left(t_{m}\right)-\nabla\left(p_{m-1}+\frac{4}{3} q_{m-1}-\frac{1}{3} q_{m-2}\right) .
$$

It was shown by Guermond and Shen that this splitting system has the following accuracy for sufficiently smooth $\boldsymbol{u}$ and $p$ : While the errors of predictor velocity and corrected velocity in $l^{2}\left(0, T ; L^{2}\right)$ norm at discrete time points are of order $k^{2}$, in $l^{2}\left(0, T ; H^{1}\right)$ norm they are of order $k^{3 / 2}$. The pressure error in $l^{2}\left(0, T ; L^{2}\right)$ norm is of order $k^{3 / 2}$ as well.

The pressure correction method stated above includes a projection of the velocity predictor $\tilde{\boldsymbol{u}}_{m}$ to a divergence free velocity $\boldsymbol{u}_{m}:=\Pi_{0} \tilde{\boldsymbol{u}}_{m}$. The projection $\Pi_{0}$ is given as $L^{2}$-projection

$$
\Pi_{0}: L^{2}(\Omega)^{d} \rightarrow \boldsymbol{H}_{0}(\Omega)
$$

on the subspace of divergence free functions (and vanishing normal traces on $\partial \Omega$ ):

$$
\boldsymbol{H}_{0}(\Omega):=\left\{\boldsymbol{u} \in L^{2}(\Omega)^{d}: \operatorname{div} \boldsymbol{u}=0 \text { in } \Omega, \boldsymbol{u} \cdot \boldsymbol{n}=0 \text { on } \partial \Omega\right\} .
$$




\section{Local Pressure Updates}

\subsection{Decomposition into subdomains}

The aim of this paper is to replace this $L^{2}$-projection by some local $L^{2}$-projection with respect to subdomains $\Omega_{i} \subset \Omega$. The advantage will be that the elliptic problem for the pressure update $(2.7)$ is replaced by a number of local Poisson problems. To this end we consider a non-overlapping decomposition of $\Omega$ :

$$
\begin{aligned}
& \bar{\Omega}=\bigcup_{i=1}^{N} \bar{\Omega}_{i} \quad \text { with } \quad \Omega_{i} \cap \Omega_{j}=\emptyset \text { for } 1 \leq i \neq j \leq N ; \\
& \widetilde{\boldsymbol{H}}_{0}\left(\Omega_{i}\right):=\left\{\boldsymbol{u} \in L^{2}\left(\Omega_{i}\right)^{d}: \operatorname{div} \boldsymbol{u}=0 \text { in } \Omega_{i}\right\} .
\end{aligned}
$$

Let

$$
P_{i}: \quad L^{2}(\Omega)^{d} \rightarrow \widetilde{\boldsymbol{H}}_{0}\left(\Omega_{i}\right)
$$

be a projection of the form in (3.2). The idea of the local pressure correction is to define $\boldsymbol{u}_{m}$ instead of Step 4 by the local definitions

$$
\left.\boldsymbol{u}_{m}\right|_{\Omega_{i}}:=P_{i} \tilde{\boldsymbol{u}}_{m}
$$

Note that the $P_{i}$ have their arguments in $L^{2}(\Omega)^{d}$ and not in $L^{2}\left(\Omega_{i}\right)^{d}$. Hence, we allow for certain global contributions. However, we will see later that the global contribution will be numerically much less costly than the original scheme. Moreover, $\left(I-P_{i}\right) \tilde{\boldsymbol{u}}_{m}$ will be a gradient,

$$
\left(I-P_{i}\right) \tilde{\boldsymbol{u}}_{m}=\frac{2 k}{3} \nabla \phi_{m}^{i}
$$

Hence, on each subdomain $\Omega_{i}$ the modified Step 2 becomes:

Step 2*: Determine $\left(I-P_{i}\right) \tilde{\boldsymbol{u}}_{m}=\frac{2 k}{3} \nabla \phi_{m}^{i}$ on each subdomain $i \in\{1, \ldots, N\}$.

Step $3^{*}$ : Pressure update

$$
\begin{aligned}
q_{m} & :=\sum_{i=1}^{N} \phi_{m}^{i}, \\
p_{m} & :=p_{m-1}+q_{m}-\nu \operatorname{div} \tilde{\boldsymbol{u}}_{m} .
\end{aligned}
$$

The new velocity is obtained as before in Step 4 by

$$
\boldsymbol{u}_{m}=\tilde{\boldsymbol{u}}_{m}-\frac{2 k}{3} \nabla q_{m}
$$

which corresponds to

$$
\left.\boldsymbol{u}_{m}\right|_{\Omega_{i}}=P_{i} \tilde{\boldsymbol{u}}_{m}=\tilde{\boldsymbol{u}}_{m}-\left(I-P_{i}\right) \tilde{\boldsymbol{u}}_{m}=\tilde{\boldsymbol{u}}_{m}-\frac{2 k}{3} \nabla \phi_{m}^{i}
$$

The way to obtain $\phi_{m}^{i}$ is not yet specified. In the next section we identify certain properties to ensure the correct error asymptotic in terms of the time step $k$. Moreover, we give one particular example in Section 5 for a realization of $\phi_{m}^{i}$ and $P_{i}$. 


\section{A Priori Error Estimate}

\subsection{Required assumption on the projection}

By $P$ we denote the global projector applied to the predictor velocity, i.e.,

$$
P: \boldsymbol{V} \rightarrow \widetilde{\boldsymbol{H}}_{0},\left.\quad P \boldsymbol{u}\right|_{\Omega_{i}}:=P_{i} \boldsymbol{u}
$$

with the space

$$
\widetilde{\boldsymbol{H}}_{0}:=\left\{\boldsymbol{v} \in L^{2}(\Omega):\left.\boldsymbol{v}\right|_{\Omega_{i}} \in \widetilde{\boldsymbol{H}}_{0}\left(\Omega_{i}\right), \quad 1 \leq i \leq N\right\}
$$

The functions $\boldsymbol{u} \in \widetilde{\boldsymbol{H}}_{0}$ may have non-vanishing jumps in normal directions across the subdomain boundaries

$$
\begin{aligned}
\|\boldsymbol{u} \cdot \boldsymbol{n}(x)\|:=\lim _{\epsilon \rightarrow 0}((\boldsymbol{u} \cdot \boldsymbol{n})(x+\epsilon \boldsymbol{n})-(\boldsymbol{u} \cdot \boldsymbol{n})(x-\epsilon \boldsymbol{n})) & & \text { for } x \in \partial \Omega_{i} \cap \Omega, \\
\|\boldsymbol{u} \cdot \boldsymbol{n}(x)\|:=(\boldsymbol{u} \cdot \boldsymbol{n})(x) & & \text { for } x \in \partial \Omega .
\end{aligned}
$$

Furthermore, let us use the notation $\Gamma$ for the union of all inner and outer subdomain boundaries:

$$
\Gamma:=\bigcup_{i=1}^{N} \partial \Omega_{i} .
$$

We will need the following assumption in order to show that the modified scheme (Step 1, Step $2^{*}$, Step $3^{*}$ and Step 4) leads to the same order of accuracy with respect to the time step as the original scheme (Step 1, Step 2, Step 3 and Step 4):

Assumption 4.1. The projections $P_{i}$ in (4.1) are mappings $P_{i}: H^{1}\left(\Omega_{i}\right) \rightarrow H^{1}\left(\Omega_{i}\right)$, and $P$ in (4.1) allows for a smallness property for the jumps of the gradients into normal direction across subdomain boundaries, i.e. for all $1 \leq i \leq N$ hold

$$
\|[P \boldsymbol{u} \cdot \boldsymbol{n}]\|_{L^{2}\left(\partial \Omega_{i}\right)} \leq c k^{3 / 2} \quad \forall \boldsymbol{u} \in H_{0}^{1}(\Omega)^{d},
$$

with a constant $c$ independent of the time step $k$ (but dependent on $\boldsymbol{u}$ ).

\subsection{Inverse Stokes operator}

In the following Lemma we address the inverse Stokes operator $S: H^{-1}(\Omega)^{d} \rightarrow \boldsymbol{V}:=$ $H_{0}^{1}(\Omega)^{d}$ given as the solution $S(\mathbf{f}) \in \boldsymbol{V}$ of $(\nabla S(\mathbf{f}), \nabla \boldsymbol{\phi})-(r, \operatorname{div} \boldsymbol{\phi})=\langle\mathbf{f}, \boldsymbol{\phi}\rangle$ for all $\boldsymbol{\phi} \in \boldsymbol{V}$ for a suitable $r \in Q:=L_{0}^{2}(\Omega)$, and $(\operatorname{div} S(\mathbf{f}), \xi)=0$ for all $\xi \in Q$. This lemma is an adaption of a similar result proved in [5]:

Lemma 4.1. Let $\Omega \subset \mathbb{R}^{d}$ be convex or $C^{2}$-bounded. For all $\boldsymbol{v} \in \boldsymbol{V}$ and all $0<\gamma<1$ it holds for $\boldsymbol{v}^{*}:=P \boldsymbol{v} \in \widetilde{\boldsymbol{H}}_{0}$ :

$$
(\nabla S(\boldsymbol{v}), \nabla \boldsymbol{v}) \geq(1-\gamma)\|\boldsymbol{v}\|_{L^{2}(\Omega)}^{2}-c(\gamma, \boldsymbol{v})\left(\left\|\boldsymbol{v}-\boldsymbol{v}^{*}\right\|_{L^{2}(\Omega)}^{2}+k^{3}\right)
$$

Proof. Firstly, we directly see that

$$
(\nabla S(\boldsymbol{v}), \nabla \boldsymbol{v})=(r, \operatorname{div} \boldsymbol{v})+\|\boldsymbol{v}\|_{L^{2}(\Omega)}^{2} .
$$


Secondly, the first term on the right hand side can be bounded as follows by integration by parts on each subdomain and for still arbitrary $\boldsymbol{v}^{*} \in \widetilde{\boldsymbol{H}}_{0}$ :

$$
\begin{aligned}
(r, \operatorname{div} \boldsymbol{v}) & =\sum_{i=1}^{N}(r, \operatorname{div} \boldsymbol{v})_{\Omega_{i}}=\sum_{i=1}^{N}\left(r, \operatorname{div}\left(\boldsymbol{v}-\boldsymbol{v}^{*}\right)\right)_{\Omega_{i}} \\
& =\sum_{i=1}^{N}\left(-\left(\nabla r, \boldsymbol{v}-\boldsymbol{v}^{*}\right)_{\Omega_{i}}-\int_{\partial \Omega_{i}} r \boldsymbol{v}^{*} \cdot \boldsymbol{n} d s\right) .
\end{aligned}
$$

Due to the regularity $\boldsymbol{v} \in \boldsymbol{V}$ we know that $r \in H^{2}(\Omega)$ is continuous in the interior of $\Omega$. Now we use a representation in terms of the jumps across interior subdomain boundaries $\Gamma$ and apply the trace theorem:

$$
\begin{aligned}
\sum_{i=1}^{N} \int_{\partial \Omega_{i}} r \boldsymbol{v}^{*} \cdot \boldsymbol{n} d s & =\int_{\Gamma} r\left\|\boldsymbol{v}^{*} \cdot \boldsymbol{n}\right\| d s \\
& \left.\left.\leq\|r\|_{L^{2}(\Gamma)}\|\| \boldsymbol{v}^{*} \cdot \boldsymbol{n}\right]\|\|_{L^{2}(\Gamma)} \leq c\|\nabla r\|_{L^{2}(\Omega)}\|\| \boldsymbol{v}^{*} \cdot \boldsymbol{n}\right]\|\|_{L^{2}(\Gamma)} .
\end{aligned}
$$

Now, we take the particular choice $\boldsymbol{v}^{*}:=P \boldsymbol{v}$. By Assumption 4.1 we obtain

$$
\int_{\Gamma} r \boldsymbol{v}^{*} \cdot \boldsymbol{n} d s \leq c k^{3 / 2}\|\nabla r\|_{L^{2}(\Omega)},
$$

with $c=c(\boldsymbol{v})$. Using this in the equation above yields

$$
(r, \operatorname{div} \boldsymbol{v}) \leq\|\nabla r\|_{L^{2}(\Omega)}\left(\left\|\boldsymbol{v}-\boldsymbol{v}^{*}\right\|_{L^{2}(\Omega)}+c k^{3 / 2}\right) .
$$

Because of $\|\nabla r\|_{L^{2}(\Omega)} \leq c\|\boldsymbol{v}\|_{L^{2}(\Omega)}$ we arrive at (4.2).

\subsection{A priori $L^{2}$-estimate for the time series}

The error estimate takes the focus on the errors with respect to the discrete time points $t_{m}$ with $t_{m}=m k$ to end time $T$ and time step size $k$. Therefore, we use capital letters for the notation of the finite time series:

$$
\boldsymbol{U}^{k}:=\left(\boldsymbol{u}_{0}, \boldsymbol{u}_{1}, \ldots, \boldsymbol{u}_{n}\right)
$$

with $n=T / k$. This notation is used for the predictor velocity, the projected velocity and the pressure, i.e. $\tilde{\boldsymbol{U}}^{k}, \boldsymbol{U}^{k}$ and $P^{k}$. The following theorem makes a statement with respect to the norm

$$
\left\|U^{k}\right\|_{l^{2}\left(L^{2}\right)}:=\left(k \sum_{j=1}^{n}\left\|u_{j}\right\|_{L^{2}(\Omega)}^{2}\right)^{1 / 2} .
$$

Theorem 4.1. We assume that Assumption 4.1 holds and the solution of (2.1)-(2.4) is suffciently smooth in space and time. Then the time-discrete solution $\left(\boldsymbol{u}^{k}, p^{k}\right)$ of Step $1,2^{*}, 3^{*}, 4$ with time-step $k>0$ satisfies the following estimates:

$$
\left\|\boldsymbol{U}-\boldsymbol{U}^{k}\right\|_{l^{2}\left(L^{2}\right)}+\left\|\boldsymbol{U}-\tilde{\boldsymbol{U}}^{k}\right\|_{l^{2}\left(L^{2}\right)} \leq c k^{2},
$$

with constant $c$ independent of the time step $k$ (but dependent on $\boldsymbol{u}$ ). 
Proof. The proof follows the lines of Guermond and Shen [4]. The only modification is that the computation of $\boldsymbol{u}_{m}$ and $p_{m}$ is different. Because the original proof is very technical and involved, we only concentrate here on those steps which have to be adapted for the scheme Step $1,2^{*}, 3^{*}, 4$. The rest follows exactly the lines of [4]. The parts of the proof where Step 2 and 3 enter are the following ones:

- A bound of the form $\left\|\tilde{\boldsymbol{U}}^{k}-\boldsymbol{U}^{k}\right\|_{l^{2}\left(L^{2}\right)} \leq c k^{2}$ is required (cf. proof Lemma 4.1 in [4]). This is still valid, because on each subdomain we have the equality

$$
\left.\left(\boldsymbol{u}_{m}-\tilde{\boldsymbol{u}}_{m}\right)\right|_{\Omega_{i}}=-\frac{2 k}{3} \nabla \phi_{m}^{i}
$$

and due to Step $3^{*}$ :

$$
\left\|\nabla \phi_{m}^{i}\right\|_{l^{2}\left(L^{2}\left(\Omega_{i}\right)\right)} \leq\left\|\nabla\left(\delta_{k} E_{p, m}+\nu \operatorname{div} \tilde{\boldsymbol{u}}_{m}\right)\right\|_{l^{2}\left(L^{2}\right)},
$$

with the series $\delta_{k} E_{p, m}:=\left(p_{m-1}-p_{m}\right)$ for $1 \leq m \leq n$. The divergence of the predictor is bounded as in the original scheme by $\left\|\nabla\left(\operatorname{div} \tilde{\boldsymbol{u}}_{m}\right)\right\|_{l^{2}\left(L^{2}\right)} \leq c k$. Hence, we still have to check that $\left\|\nabla \delta_{k} E_{p, m}\right\|_{l^{2}\left(L^{2}\right)} \leq c k$. However, proving this is identical to the original scheme, because it is just a consequence of Step 1 and Step 4 (see Section 4.2 in [4]).

- The following property of the inverse Stokes operator $S$ is used by Guermond and Shen (Lemma 2.1 of $[4])$ :

$$
(\nabla S(\boldsymbol{v}), \nabla \boldsymbol{v}) \geq(1-\gamma)\|\boldsymbol{v}\|_{L^{2}(\Omega)}^{2}-c(\gamma)\left\|\boldsymbol{v}-\boldsymbol{v}^{*}\right\|_{L^{2}(\Omega)}^{2} \quad \forall \boldsymbol{v}^{*} \in \boldsymbol{H}_{0}
$$

Since our $\boldsymbol{u}_{m}$ is in $\widetilde{\boldsymbol{H}}_{0}$ rather than in $\boldsymbol{H}_{0}$, we have to use Lemma 4.1 , leading to

$$
\left\|\boldsymbol{U}-\tilde{\boldsymbol{U}}^{k}\right\|_{l^{2}\left(L^{2}\right)} \leq c\left\|\tilde{\boldsymbol{U}}^{k}-\boldsymbol{U}^{k}\right\|_{l^{2}\left(L^{2}\right)}+c k^{2} .
$$

Together with $\left\|\tilde{\boldsymbol{U}}^{k}-\boldsymbol{U}^{k}\right\|_{l^{2}\left(L^{2}\right)} \leq c k^{2}$ (see above) we arrive at

$$
\left\|\boldsymbol{U}-\tilde{\boldsymbol{U}}^{k}\right\|_{l^{2}\left(L^{2}\right)} \leq c k^{2} .
$$

Note that the $\boldsymbol{u}$-dependence of the constant $c$ does not harm the asymptotic dependency $\mathcal{O}\left(k^{2}\right)$

\subsection{A priori $H^{1}$-estimate for the time series}

Now, we address the norm

$$
\left\|U^{k}\right\|_{l^{2}\left(H^{1}\right)}:=\left(k \sum_{j=1}^{n} \sum_{i=1}^{N}\left\|u_{j}\right\|_{H^{1}\left(\Omega_{i}\right)}^{2}\right)^{1 / 2} .
$$

Assumption 4.2. The projections $P_{i}$ in (3.1) are $L^{2}$ - and $H^{1}$-stable, i.e. for all $1 \leq i \leq N$ hold

$$
\begin{array}{rlrl}
\left\|P_{i} \boldsymbol{u}\right\|_{L^{2}\left(\Omega_{i}\right)^{d}} & \leq c_{i}\|\boldsymbol{u}\|_{L^{2}(\Omega)^{d}} & \forall \boldsymbol{u} \in L^{2}(\Omega)^{d}, \\
\left\|\nabla P_{i} \boldsymbol{u}\right\|_{L^{2}\left(\Omega_{i}\right)^{d \times d}} \leq c_{i}\|\boldsymbol{u}\|_{H^{1}(\Omega)^{d}} & \forall \boldsymbol{u} \in H^{1}(\Omega)^{d},
\end{array}
$$

with constants $c_{i}$ independent of the time step $k$ (but dependent on $\Omega_{i}$ ). 
Theorem 4.2. We assume that Assumptions 4.1 and 4.2 hold, and that the solution of (2.1)(2.4) is sufficiently smooth in space and time. Then the time-discrete solution $\left(\boldsymbol{u}^{k}, p^{k}\right)$ of Step $1,2^{*}, 3^{*}, 4$ with time-step $k>0$ satisfies the following estimates:

$$
\left\|\boldsymbol{U}-\boldsymbol{U}^{k}\right\|_{l^{2}\left(H^{1}\right)}+\left\|\boldsymbol{U}-\tilde{\boldsymbol{U}}^{k}\right\|_{l^{2}\left(H^{1}\right)} \leq c k^{3 / 2}
$$

with constant $c$ independent of the time step $k$, but possibly dependent on the partition of $\Omega$ and $\boldsymbol{u}$.

Proof. The proof for the spatial $H^{1}$-error for the original scheme requires that $\boldsymbol{u}_{m}$ is a $L^{2}$ and $H^{1}$-stable projection of the predictor velocity $\tilde{\boldsymbol{u}}_{m}$ (cf. Lemma 4.4 in [4]). This is also the case for the local correction scheme, $\left.\boldsymbol{u}_{m}\right|_{\Omega_{i}}=P_{i} \tilde{\boldsymbol{u}}_{m}$, because of Assumption 4.2. This assumption only ensures $H^{1}$-stability with respect to the subdomains $\Omega_{i}$. That is why the norm (4.6) only includes the gradients within these subdomains and not across the interior boundaries $\partial \Omega_{i} \cap \partial \Omega_{j}(i \neq j)$.

\section{Realization of the projections $P_{i}$}

In this section we discuss possibilities to realize the projections $P_{i}$ in (3.1). One possibility is to take $P_{i}$ as the restriction of the $L^{2}$-projection $\Pi_{0}: L^{2}(\Omega)^{d} \rightarrow \boldsymbol{H}_{0}$ to the subdomain $\Omega_{i}$. This would lead to $P=\Pi_{0}$ and, hence, to the original scheme. Here, we want to define projections which are numerically less expensive and suitable to parallelization. However, we are not completely free in the choice of the $P_{i}$, because Assumptions 4.1 and 4.2 should be valid.

The computation of $P_{i}$ consists of two steps for $\tilde{\boldsymbol{u}}:=\tilde{\boldsymbol{u}}_{m}$ :

First step: At first, we compute a coarse grid approximation $q_{H}$ of the pressure correction $q_{m}$ :

$$
\begin{aligned}
-\Delta q_{H} & \approx-\frac{3}{2 k} \operatorname{div} \tilde{\boldsymbol{u}} & & \text { in } \Omega, \\
\nabla q_{H} \cdot \boldsymbol{n} & =0 & & \text { on } \partial \Omega .
\end{aligned}
$$

Here we write ' $\approx$ ', because it is only an approximation, so that we don't have point-wise equality here. This 'equation' has to be understood in weak formulations for test functions of a finite dimensional space (finite elements) for a large spatial mesh size $H>0$. The particular way to solve (5.1)-(5.2) is very flexible, we only make the following assumption:

Assumption 5.1. The discretization of (5.1)-(5.2) is designed in such a way that $q_{H}$ is welldefined and the following properties hold, if $q_{m} \in H^{2}(\Omega)$ :

$$
\begin{aligned}
\left\|\nabla\left(q_{m}-q_{H}\right)\right\|_{L^{2}(\Omega)} & \leq C H\left\|q_{m}\right\|_{H^{2}(\Omega)}, \\
\left\|\nabla q_{H}\right\|_{L^{2}(\Omega)}+\sum_{T \in \mathcal{T}_{H}}\left|q_{H}\right|_{H^{2}(T)}^{2}+\sum_{e \in \mathcal{E}_{H}}\left\|\left|\left[\nabla q_{H} \cdot \boldsymbol{n}\right]\right|\right\|_{L^{1}(e)} & \leq C k^{-1}\|\operatorname{div} \tilde{\boldsymbol{u}}\|_{L^{2}(\Omega)},
\end{aligned}
$$

where $\mathcal{T}_{H}$ denotes the set of all elements, and $\mathcal{E}_{H}$ the set of all inner edges.

Second step: In the second step we solve local Poisson problems:

$$
\begin{aligned}
-\Delta \phi_{m}^{i} & =-\frac{3}{2 k} \operatorname{div} \tilde{\boldsymbol{u}} & & \text { in } \Omega_{i}, \\
\nabla \phi_{m}^{i} \cdot \boldsymbol{n} & =\Pi_{H}\left(\nabla q_{H} \cdot \boldsymbol{n}\right)+g_{i} & & \text { on } \Gamma_{i}:=\partial \Omega_{i} \backslash \partial \Omega, \\
\nabla \phi_{m}^{i} \cdot \boldsymbol{n} & =0 & & \text { on } \partial \Omega_{i} \cap \partial \Omega,
\end{aligned}
$$


with

$$
g_{i}:=\left|\Gamma_{i}\right|^{-1} \int_{\Gamma_{i}}\left(\frac{3}{2 k} \tilde{\boldsymbol{u}}_{m} \cdot \boldsymbol{n}-\Pi_{H}\left(\nabla q_{H} \cdot \boldsymbol{n}\right)\right) d s
$$

and a $L^{2}$-projection to a finite dimensional space $S_{H}$

$$
\Pi_{H}: L^{2}(\Omega) \rightarrow S_{H} \in C(\bar{\Omega})
$$

so that $\Pi_{H}\left(\nabla q_{H} \cdot \boldsymbol{n}\right)$ is continuous on interfaces $\Gamma_{i}$ (see Section 6 ). We assume that $\Pi_{H}$ is $H^{1}$-stable which is usually the case, see Bramble et al. [1]. The projection of the gradient $\Pi_{H}\left(\nabla q_{H} \cdot \boldsymbol{n}\right)$ in the right hand side of the Neumann data can be considered as the principal part, whereas the $g_{i}$ are corrections in order to ensure the compatibility property

$$
\int_{\Gamma_{i}}\left(\Pi_{H}\left(\nabla q_{H} \cdot \boldsymbol{n}\right)+g_{i}\right) d S=\frac{3}{2 k} \int_{\Omega_{i}} \operatorname{div} \tilde{\boldsymbol{u}} d x, \quad 1 \leq i \leq N .
$$

This ensures the solvability and uniqueness of the local Poisson problems (5.3)-(5.4). The projection $I-P_{i}$ is now given by

$$
\left(I-P_{i}\right) \tilde{\boldsymbol{u}}_{m}:=\frac{2 k}{3} \nabla \phi_{m}^{i} .
$$

In order to validate that $P_{i}$ really maps into $\widetilde{\boldsymbol{H}}_{0}\left(\Omega_{i}\right)$, we deduce

$$
\left.\boldsymbol{u}_{m}\right|_{\Omega_{i}}=P_{i} \tilde{\boldsymbol{u}}_{m}=\left.\tilde{\boldsymbol{u}}_{m}\right|_{\Omega_{i}}-\left(I-P_{i}\right) \tilde{\boldsymbol{u}}_{m}=\left.\tilde{\boldsymbol{u}}_{m}\right|_{\Omega_{i}}-\frac{2 k}{3} \nabla \phi_{m}^{i},
$$

which shows that

$$
\left.\operatorname{div} \boldsymbol{u}_{m}\right|_{\Omega_{i}}=\left.\operatorname{div} \widetilde{\boldsymbol{u}}_{m}\right|_{\Omega_{i}}-\frac{2 k}{3} \Delta \phi_{m}^{i}=0 .
$$

This modified scheme has some advantages compared to the original one:

1. The Poisson problems (5.3)-(5.4) can be solved in parallel without any sophisticated parallel solver. These $N$ individual problems are decoupled from each other so that the communication costs are minimal.

2. If the partition consists of sufficiently small subdomain $\Omega_{i}$, the discretization of the local problems (5.3)-(5.4) may lead to very small systems which can be efficiently and robustly solved by a simple direct solver. Therefore, no sophisticated elliptic solver (e.g. multigrid) is needed to handle the large condition numbers of Poisson problems.

3. Solving the local problems (5.3)-(5.4) are usually more memory efficient on modern CPUs with fast cache memories, because all needed data can be stored in the cache, if $N$ is large enough.

4. In certain applications with (very) small time steps, the momentum equation (2.5) is often solved in an explicit way without any need for an implicit solver. In this case, only an elliptic coarse grid problem for $q_{H}$ and several small systems have to be solved in the entire algorithm (Step 1, 2*, 3 and 4 ). 


\subsection{Check of Assumption 4.1}

In order to show that Assumption 4.1 is valid, if Assumption 5.1 holds, we make use of the following property shown in [4]:

$$
\left\|\operatorname{div} \tilde{\boldsymbol{u}}_{m}\right\|_{L^{2}(\Omega)} \leq c k^{3 / 2} .
$$

This property is also true for our algorithm, because Step 1 is the same, i.e. the procedure to determine $\tilde{\boldsymbol{u}}_{m}$ is the same as the original scheme. Note that the constant $c$ may depend on $\nabla \tilde{\boldsymbol{u}}_{m}$. However, the explicit dependency of the constant is not specified in [4], and is also not addressed in this work.

Lemma 5.1. Assumption 5.1 in combination with a $H^{1}$-stable $L^{2}$-projection $\Pi_{H}$ ensures the property in Assumption 4.1.

Proof. Step A: We firstly show that Assumption 5.1 implies that for all $1 \leq i \leq N$,

$$
\left|g_{i}\right| \leq c k^{1 / 2}
$$

with $c=c\left(\nabla \boldsymbol{u}_{m}\right)$ and $\tilde{\boldsymbol{u}}:=\tilde{\boldsymbol{u}}_{m}$. For the real numbers $g_{i}$ we derive

$$
\begin{aligned}
\left|g_{i}\right| & =\left|\Gamma_{i}\right|^{-1}\left|\int_{\Gamma_{i}}\left(\frac{3}{2 k} \tilde{\boldsymbol{u}} \cdot \boldsymbol{n}-\Pi_{H}\left(\nabla q_{H} \cdot \boldsymbol{n}\right)\right) d s\right| \\
& =\left|\Gamma_{i}\right|^{-1}\left|\int_{\Gamma_{i}}\left(\nabla q_{m} \cdot \boldsymbol{n}-\Pi_{H}\left(\nabla q_{H} \cdot \boldsymbol{n}\right)\right) d s\right| \\
& =\left|\Gamma_{i}\right|^{-1}\left(j\left(q_{m}\right)-j_{\Pi}\left(q_{H}\right)\right),
\end{aligned}
$$

with the functionals

$$
j(q):=\int_{\Gamma_{i}} \nabla q \cdot \boldsymbol{n} d s \quad \text { and } \quad j_{\Pi}(q):=\int_{\Gamma_{i}} \Pi_{H}(\nabla q \cdot \boldsymbol{n}) d s .
$$

By assumption, the projection $\Pi_{H}$ is $L^{2}$-stable and $H^{1}$-stable:

$$
\begin{array}{ll}
\left\|\Pi_{H} v\right\|_{L^{2}(\Omega)} \leq C_{1}\|v\|_{L^{2}(\Omega)} & \forall v \in L^{2}(\Omega), \\
\left\|\Pi_{H} v\right\|_{H^{1}(\Omega)} \leq C_{2}\|v\|_{H^{1}(\Omega)} & \forall v \in H^{1}(\Omega) .
\end{array}
$$

This implies $j_{\Pi} \in H^{-1}(\Omega)$ and $\left\|j_{\Pi}\right\|_{H^{-1}(\Omega)} \leq C_{2} H^{-1}$, because of the trace theorem and an inverse estimate $\left(q \in H^{1}(\Omega)\right)$ :

$$
\begin{aligned}
\left|j_{\Pi}(q)\right| & \leq c_{\Gamma_{i}}\left\|\Pi_{H}(\nabla q \cdot \boldsymbol{n})\right\|_{H^{1}(\Omega)} \leq c_{\Gamma_{i}} c_{i n v} H^{-1}\left\|\Pi_{H}(\nabla q \cdot \boldsymbol{n})\right\|_{L^{2}(\Omega)} \\
& \leq c_{\Gamma_{i}} c_{i n v} H^{-1}\|\nabla q \cdot \boldsymbol{n}\|_{L^{2}(\Omega)} \leq c_{\Gamma_{i}} c_{i n v} C_{1} H^{-1}|q|_{H^{1}(\Omega)} .
\end{aligned}
$$

Moreover, $j-j_{\Pi} \in H^{2}(\Omega)^{\prime}$, because of the trace theorem $\left(q \in H^{2}(\Omega)\right)$

$$
\left|\left(j-j_{\Pi}\right)(q)\right| \leq c_{\Gamma_{i}}\left\|\left(I-\Pi_{H}\right)(\nabla q \cdot \boldsymbol{n})\right\|_{H^{1}(\Omega)} \leq c_{\Gamma_{i}}\left(1+C_{2}\right)\|q\|_{H^{2}(\Omega)} .
$$

This allows to bound the real numbers $\left|g_{i}\right|$ accordingly:

$$
\begin{aligned}
\left|g_{i}\right| & \leq c\left(\left|\left(j-j_{\Pi}\right)\left(q_{m}\right)\right|+\left|j_{\Pi}\left(q_{m}-q_{H}\right)\right|\right) \\
& \leq c_{\Gamma_{i}}\left(1+C_{2}\right)\left\|q_{m}\right\|_{H^{2}\left(\Omega_{i}\right)}+c_{\Gamma_{i}} c_{i n v} C_{1} H^{-1}\left\|\nabla\left(q_{m}-q_{H}\right)\right\|_{L^{2}\left(\Omega_{i}\right)} \\
& \leq c\left\|q_{m}\right\|_{H^{2}\left(\Omega_{i}\right)} \leq c k^{-1}\|\operatorname{div} \tilde{\boldsymbol{u}}\|_{L^{2}(\Omega)} \leq c k^{1 / 2},
\end{aligned}
$$


where we used (5.7) in the last inequality.

Step B: Let $\boldsymbol{u} \in H_{0}^{1}(\Omega)^{d}$ and $\Gamma:=\partial \Omega_{i} \cap \partial \Omega_{j}$ be an internal interface of two subdomains $\Omega_{i}$ and $\Omega_{j}$ with $i \neq j$. Since $\boldsymbol{u}$ has a well-defined $L^{2}$-trace on $\Gamma$, and $\Pi_{H}\left(\nabla q_{H} \cdot \boldsymbol{n}\right)$ is continuous across $\Gamma$, we have

$$
\begin{aligned}
\|[P \boldsymbol{u} \cdot \boldsymbol{n}]\|_{L^{2}(\Gamma)} & =\frac{2 k}{3}\left\|\nabla \phi_{m}^{i} \cdot \boldsymbol{n}-\nabla \phi_{m}^{j} \cdot \boldsymbol{n}\right\|_{L^{2}(\Gamma)} \\
& =\frac{2 k}{3}\left\|g_{i}-g_{j}\right\|_{L^{2}(\Gamma)} \leq \frac{2 k}{3}\left(\left|g_{i}\right|+\left|g_{j}\right|\right)|\Gamma|^{1 / 2} .
\end{aligned}
$$

Using now the result of Step A in this proof yields the assertion.

\subsection{Check of Assumption 4.2}

In order to show the $H^{1}$-stability of $P_{i}$, we first split the $L^{2}$-norm in additive way as

$$
\left\|\nabla P_{i} \widetilde{\boldsymbol{u}}\right\|_{L^{2}\left(\Omega_{i}\right)} \leq\|\nabla \widetilde{\boldsymbol{u}}\|_{L^{2}\left(\Omega_{i}\right)}+\left\|\nabla\left(I-P_{i}\right) \widetilde{\boldsymbol{u}}\right\|_{L^{2}\left(\Omega_{i}\right)} .
$$

It remains to bound $\|\nabla(I-P) \widetilde{\boldsymbol{u}}\|_{L^{2}\left(\Omega_{i}\right)}$ properly. By standard stability results for elliptic problems on convex or smooth domains we have:

$$
\begin{aligned}
\left\|\nabla\left(I-P_{i}\right) \widetilde{\boldsymbol{u}}\right\|_{L^{2}\left(\Omega_{i}\right)} & \leq c k\left\|\nabla^{2} \phi_{m}^{i}\right\|_{L^{2}\left(\Omega_{i}\right)} \\
& \leq c k\left(k^{-1}\|\operatorname{div} \tilde{\boldsymbol{u}}\|_{L^{2}\left(\Omega_{i}\right)}+\left\|\Pi_{H}\left(\nabla q_{H} \cdot \boldsymbol{n}\right)+g_{i}\right\|_{L^{2}\left(\partial \Omega_{i}\right)}\right) \\
& \leq c\|\nabla \tilde{\boldsymbol{u}}\|_{L^{2}\left(\Omega_{i}\right)}+c_{i} k\left(\left\|\Pi_{H}\left(\nabla q_{H} \cdot \boldsymbol{n}\right)\right\|_{L^{2}\left(\partial \Omega_{i}\right)}+\left|g_{i}\right|\right) .
\end{aligned}
$$

The constant $c_{i}$ may depend on $\Omega_{i}$. The trace inequality and stability of finite dimensional elliptic problems yields

$$
\begin{aligned}
\left\|\Pi_{H}\left(\nabla q_{H} \cdot \boldsymbol{n}\right)\right\|_{L^{2}\left(\partial \Omega_{i}\right)} & \leq c_{\Gamma_{i}}\left\|\Pi_{H}\left(\nabla q_{H} \cdot \boldsymbol{n}\right)\right\|_{H^{1}\left(\Omega_{i}\right)} \\
& \leq c H^{-1}\left\|\nabla q_{H}\right\|_{L^{2}\left(\Omega_{i}\right)} \leq c H^{-1} k^{-1}\|\operatorname{div} \tilde{\boldsymbol{u}}\|_{L^{2}(\Omega)} .
\end{aligned}
$$

Moreover, we already showed in the previous subsection that $\left|g_{i}\right| \leq c k^{1 / 2}\|\nabla \tilde{\boldsymbol{u}}\|_{L^{2}(\Omega)}$. Hence, we arrive at

$$
\left\|\nabla\left(I-P_{i}\right) \widetilde{\boldsymbol{u}}\right\|_{L^{2}\left(\Omega_{i}\right)} \leq c_{i}\|\nabla \tilde{\boldsymbol{u}}\|_{L^{2}(\Omega)},
$$

with $c_{i}=c_{i}\left(\Omega_{i}, H\right)$. The $L^{2}$-stability is obtained analogously using $\|\operatorname{div} \tilde{\boldsymbol{u}}\|_{H^{-1}\left(\Omega_{i}\right)} \leq\|\tilde{\boldsymbol{u}}\|_{L^{2}\left(\Omega_{i}\right)}$. Note that the factor $H^{-1}$ appearing in the stability estimates of $P_{i}$ is not critical, due to the fact that, it is only a coarse spatial mesh width.

\section{Numerical Results}

In order to compare the accuracy of the proposed method numerically, we consider the two-dimensional example presented in [4]:

$$
\boldsymbol{u}(x, y, t)=\left(\begin{array}{c}
\sin (x+t) \sin (y+t) \\
\cos (x+t) \cos (y+t)
\end{array}\right), \quad p(x, y)=\sin (x-y+t)
$$


in the unit square domain $(0,1)^{2}$ and time intervall $I:=(0, T], T=1$. The time step sizes are chosen as $k=0.1 \cdot 2^{-n}, n \in\{0, \cdots, 5\}$. We use Taylor-Hood $P_{2} / P_{1}$ finite elements. As spatial mesh size we choose $h=2^{-7}$. The elliptic coarse grid problem for $q_{H}$ is carried out by $P_{1}$-elements for the mesh size $H=8 h$. Hence, solving this problem is a factor 64 smaller (with respect to the number of unknowns) than the one for the pressure of the original pressure correction scheme. Therefore, the computation of $q_{H}$ is basically for free. The used $L^{2}$-projection $\Pi_{H}$ in Step $2^{*}$ consists of projection onto quadratic finite elements on the mesh with cell size $2 H$.

Now, let us check, if Assumption 5.1 is fulfilled. It is well known, that the $P_{1}$-discretization for the Poisson problem is well-defined and has accuracy of first order, i.e. for $q_{H} \in Q_{H}$ holds (5.3). Moreover, we have $\left|q_{H}\right|_{H^{2}(T)}=0$ for all $T \in \mathcal{T}_{H}$. The bound of $\left\|\nabla q_{H}\right\|_{L^{2}(\Omega)}$ is easily obtained by testing the discrete variational formulation diagonally. Hence, it remains to check
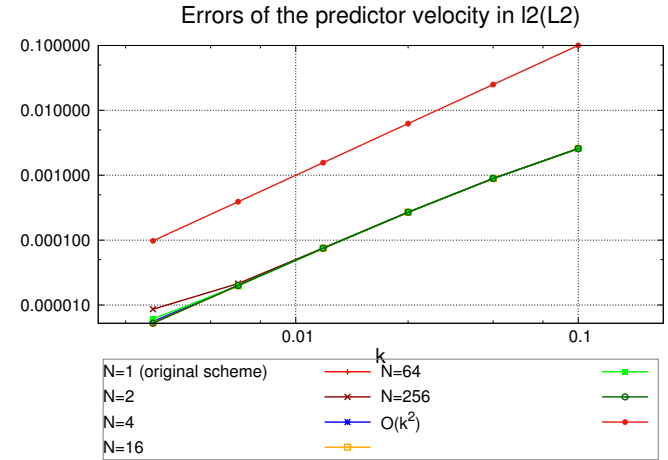

$\mathrm{N}=16$

Errors of the corrector velocity in I2(L2)

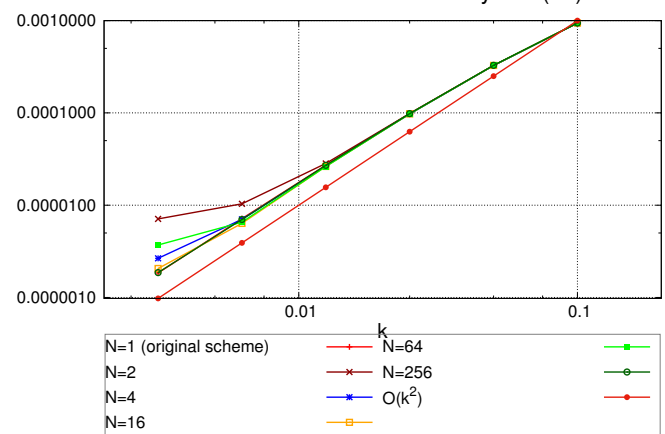

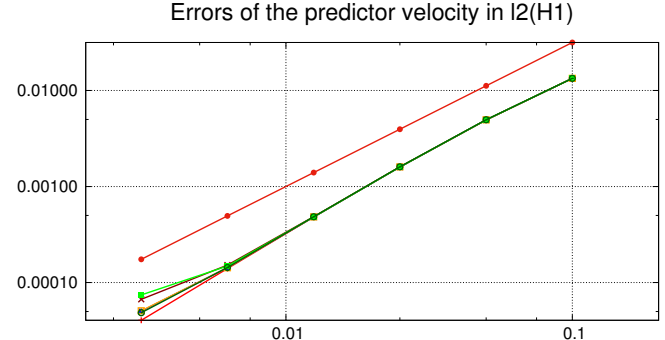

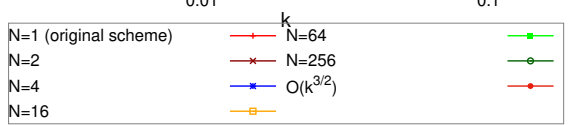

$N=16$

Errors of the pressure in I2(L2)
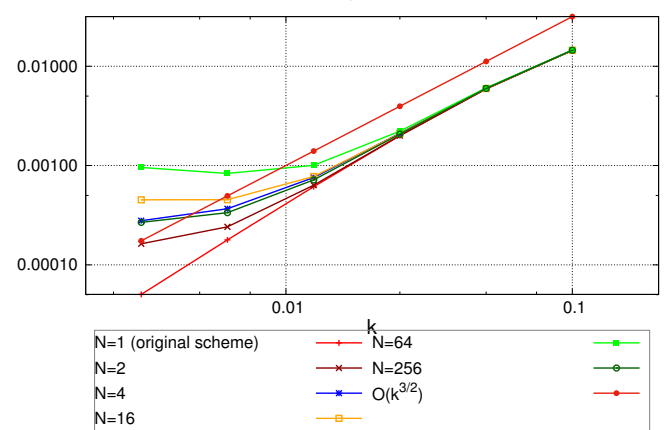

Errors of the pressure in $\mathrm{I} 2(\mathrm{H} 1)$
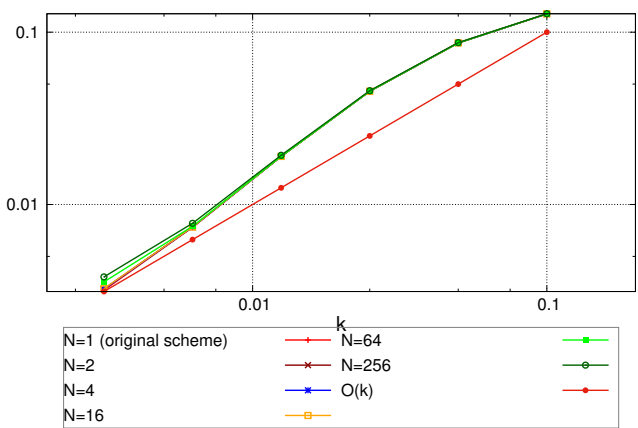

Fig. 6.1. Numerical results for $\nu=1$. 
the upper bound for the jumps across element edges. The jump $\mid\left[\nabla q_{H} \cdot \boldsymbol{n}\right]$ on an edge $e \in \mathcal{E}_{H}$ is constant. We denote this value by $\left|\left[\nabla q_{H} \cdot \boldsymbol{n}\right]\right|_{e} \in \mathbb{R}$. We have for all test functions $\varphi \in Q_{H}$

$$
\begin{aligned}
k^{-1}\left(\operatorname{div} \tilde{\boldsymbol{u}}_{m}, \varphi\right) & =\left(\nabla q_{H}, \nabla \varphi\right) \\
& =\sum_{T \in \mathcal{T}_{H}}\left(-\Delta q_{H}, \varphi\right)_{T}+\int_{\partial T} \nabla q_{H} \cdot \boldsymbol{n} \varphi d s \\
& =\frac{1}{2} \sum_{e \in \mathcal{E}_{H}} \int_{e}\left|\left[\nabla q_{H} \cdot \boldsymbol{n}\right]\right| \varphi d s=\frac{1}{2} \sum_{e \in \mathcal{E}_{H}}\left|\left[\nabla q_{H} \cdot \boldsymbol{n}\right]\right|_{e} \int_{e} \varphi d s \\
& =\frac{1}{2} \sum_{e \in \mathcal{E}_{H}}\left|\left[\nabla q_{H} \cdot \boldsymbol{n}\right]\right|_{e}|e| \varphi\left(x_{e}\right),
\end{aligned}
$$

with the midpoint $x_{e}$ of edge $e$. We choose the particular discrete test function $\varphi^{*} \in Q_{H}$ which has the values \pm 1 at $x_{e}$, according to the sign of the jumps of $q_{H}$ in normal direction, $\varphi^{*}\left(x_{e}\right):=\operatorname{sign}\left(\left|\left[\nabla q_{H} \cdot \boldsymbol{n}\right]\right|_{e}\right)$ :

$$
\begin{aligned}
\sum_{e \in \mathcal{E}_{h}}\left\|\left[\nabla q_{H} \cdot \boldsymbol{n}\right]\right\| \|_{L^{1}(e)} & =2 k^{-1}\left(\operatorname{div} \tilde{\boldsymbol{u}}_{m}, \varphi^{*}\right) \\
& \leq 2 k^{-1}\left\|\operatorname{div} \tilde{\boldsymbol{u}}_{m}\right\|_{L^{2}(\Omega)}\left\|\varphi^{*}\right\|_{L^{2}(\Omega)} \leq C k^{-1}\left\|\operatorname{div} \tilde{\boldsymbol{u}}_{m}\right\|_{L^{2}(\Omega)} .
\end{aligned}
$$

This shows that Assumption 5.1 holds.
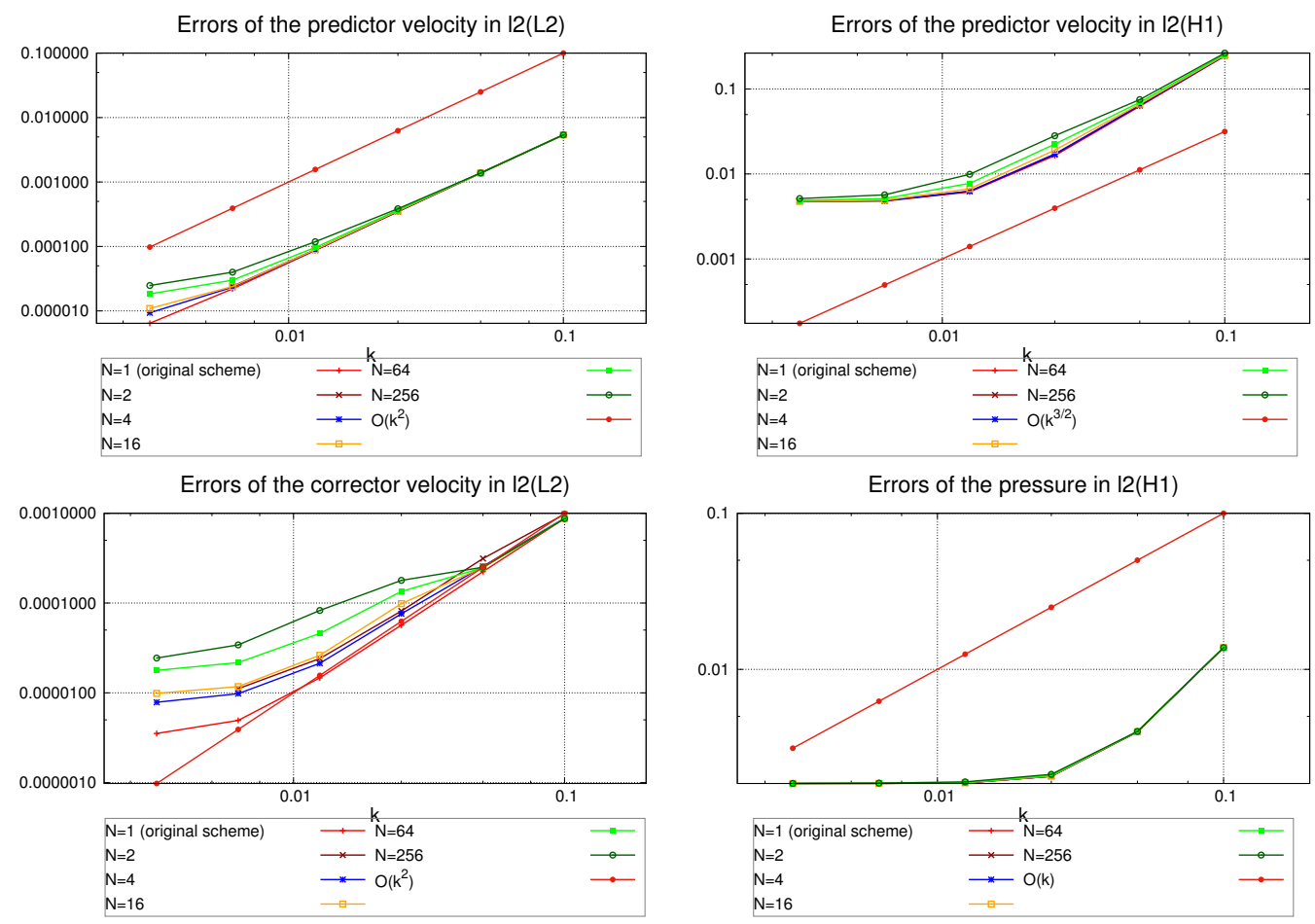

Fig. 6.2. Numerical results for $\nu=10^{-6}$. 


\subsection{Results for $\nu=1$}

In the first test we consider the Stokes problem with viscosity $\nu=1$ for an increasing number of subdomains $N \in\{1,2,4,16,64,256\}$, where $N=1$ corresponds to the original scheme without subdomains. In Fig. 6.1 we show the obtained convergence rates of $\| \boldsymbol{U}-$ $\tilde{\boldsymbol{U}}^{k}\left\|_{l^{2}\left(L^{2}\right)},\right\| \boldsymbol{U}-\tilde{\boldsymbol{U}}^{k}\left\|_{l^{2}\left(H^{1}\right)},\right\| \boldsymbol{U}-\boldsymbol{U}^{k}\left\|_{l^{2}\left(L^{2}\right)},\right\| P-P^{k} \|_{l^{2}\left(L^{2}\right)}$ and $\left\|P-P^{k}\right\|_{l^{2}\left(H^{1}\right)}$ in dependence of the time step size $k$. We see that the errors $\left\|\boldsymbol{U}-\tilde{\boldsymbol{U}}^{k}\right\|_{l^{2}\left(L^{2}\right)}$ and $\left\|\boldsymbol{U}-\tilde{\boldsymbol{U}}^{k}\right\|_{l^{2}\left(H^{1}\right)}$ are nearly independent of the number of subdomains. Only for very small time steps $k=3.125 \cdot 10^{-3}$ we observe a very small degeneration of convergence. However, this minor lack of accuracy becomes smaller with increasing number $N$ in such a way that for very high number of subdomains $(N=256)$ no difference to the original scheme can be observed. The error in the projected velocity $\left\|\boldsymbol{U}-\boldsymbol{U}^{k}\right\|_{l^{2}\left(L^{2}\right)}$ also behaves very accurate, but slightly worse for the smallest time steps, at least for a small number of subdomains. The biggest differences can be observed for the error $\left\|P-P^{k}\right\|_{l^{2}\left(L^{2}\right)}$ : The error tends to stagnation for small time steps. This can be explained by the fact that the pressure of the discrete scheme becomes discontinuous over subdomain boundaries

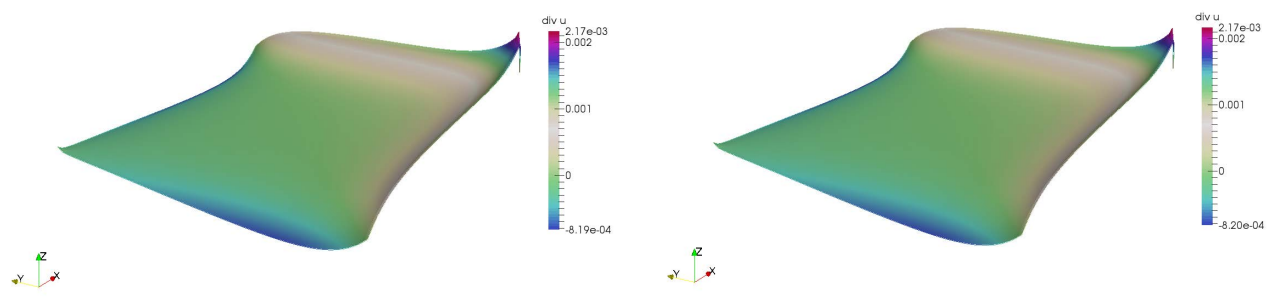

Fig. 6.3. Divergence of predictor velocity for $\nu=1$ at $T=1, d t=1.25^{-2}$. Left: original scheme, right: $N=16$.
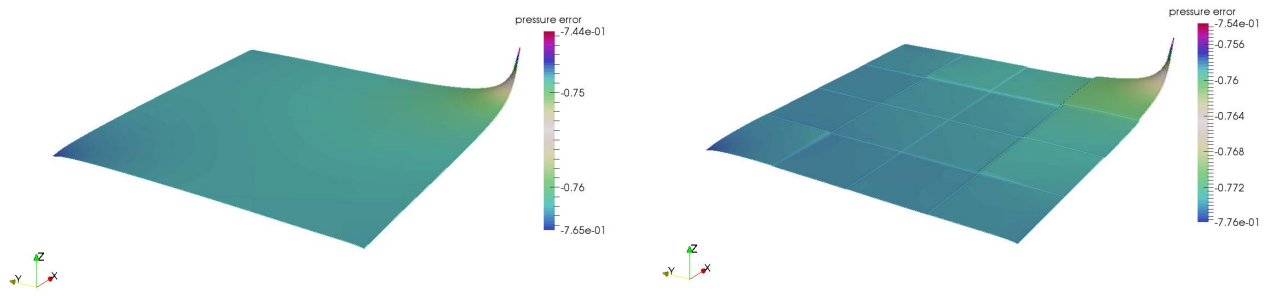

Fig. 6.4. Pressure error for $\nu=1$ at $T=1, d t=1.25^{-2}$. Left: original scheme, right: $N=16$.
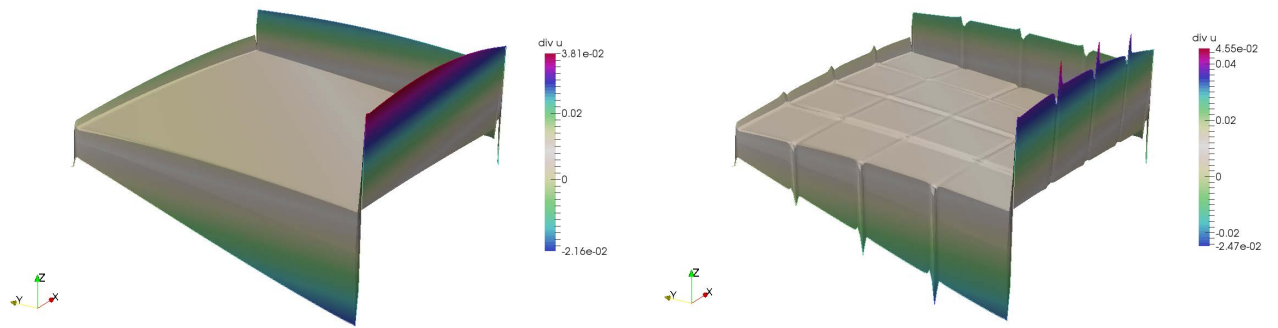

Fig. 6.5. Divergence of predictor velocity for $\nu=10^{-6}$ at $T=1, d t=1.25^{-2}$. Left: original scheme, right: $N=16$. 
and its mean value on each subdomain is arbitrary. There is no way to really determine the mean value which would correspond to the one of the global pressure. However, having a look on the error $\left\|P-P^{k}\right\|_{l^{2}\left(H^{1}\right)}$, i.e. pressure error with respect to the spatial gradient, the local

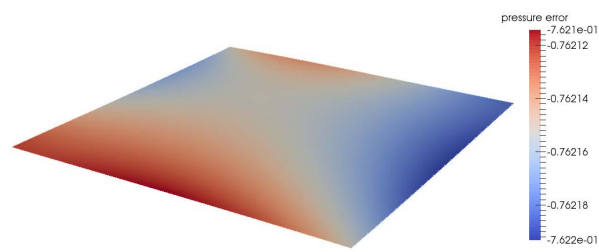

$v^{2}{ }^{2} \times$

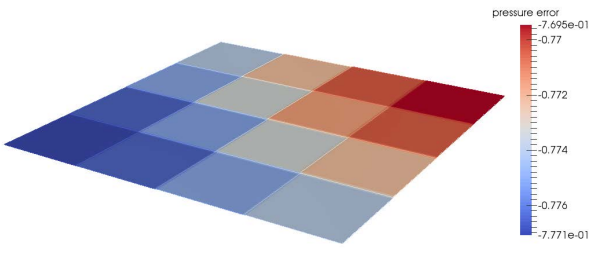

$*^{2} *$

Fig. 6.6. Pressure error for $\nu=10^{-6}$ at $T=1, d t=1.25^{-2}$. Left: original scheme, right: $N=16$.
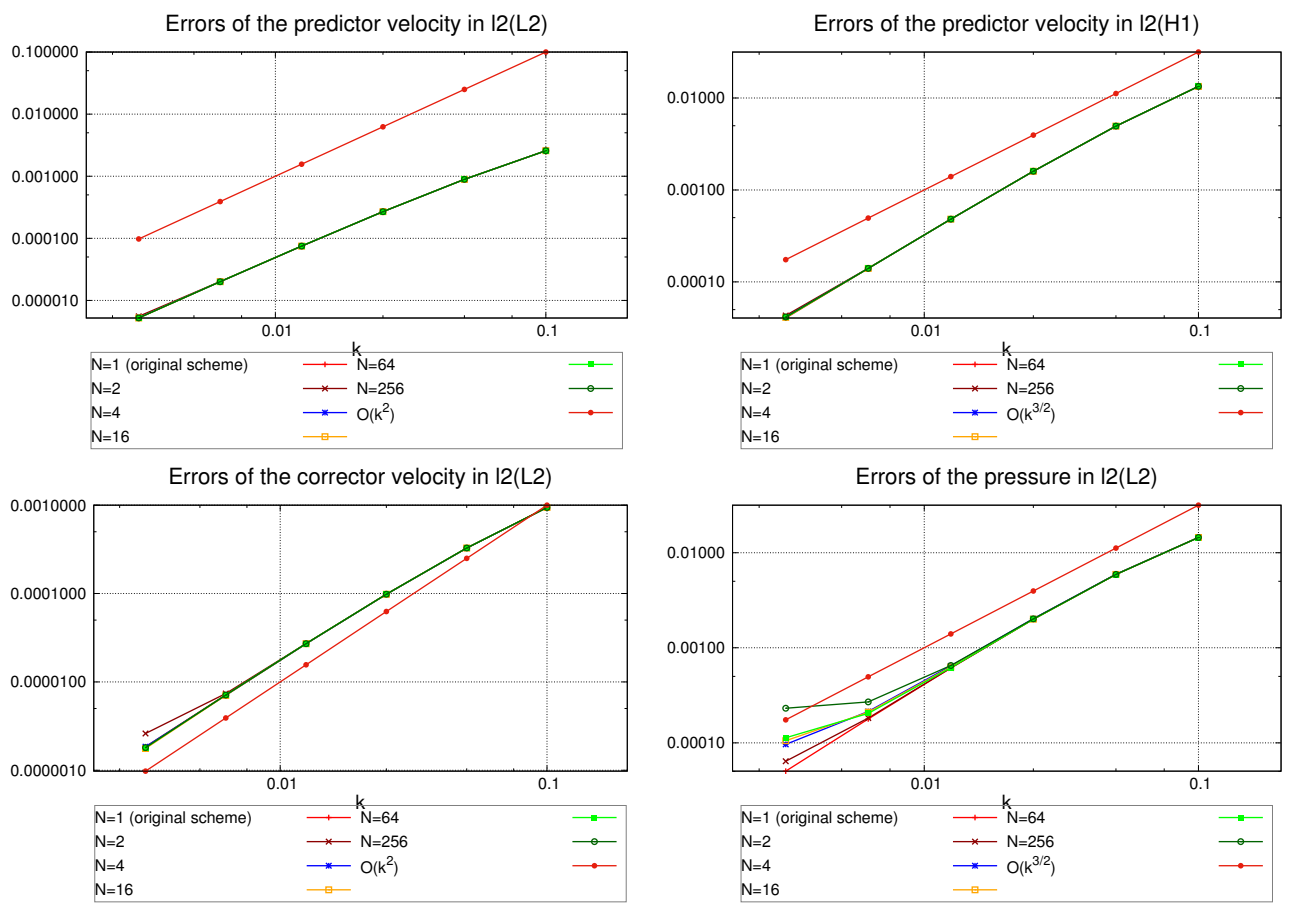

$\mathrm{N}=16$


$\mathrm{N}=16$
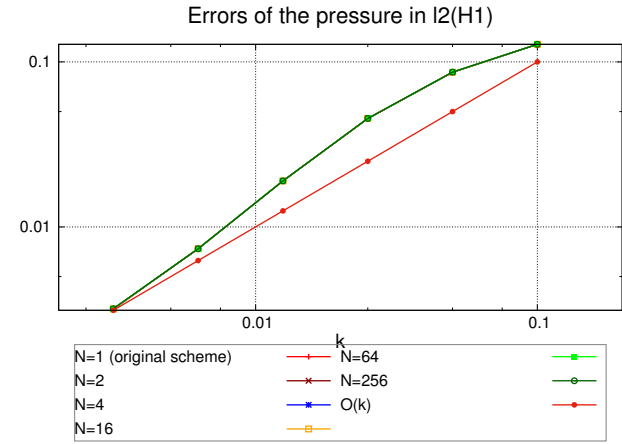

Fig. 6.7. Numerical results for $\nu=1, H=4 h$. 
pressure correction leads to the same accuracy as the original scheme, nearly independent of the number of subdomains. Employing a finer coarse grid $(H=4 h)$ leads to slightly more accurate results at small time steps, see Fig. 6.7. Moreover, in Figures 6.3 and 6.4 we present div $\widetilde{\boldsymbol{u}}(T)$ and $\left(p-p_{h}\right)(T)$ for both original scheme and local projection scheme with $N=16$.

\subsection{Results for $\nu=10^{-6}$}

In the second type of tests, we modify the viscosity to $\nu=10^{-6}$. The reason is that a weighting of the diffusive term in the Stokes equation leads to a different scaling between pressure and velocity. This is important to have a first check wether the method is suitable for Navier-Stokes equations, where we usually have to treat small values of $\nu$. The obtained results are shown in Fig. 6.2. The norm $\left\|\boldsymbol{U}-\tilde{\boldsymbol{U}}^{k}\right\|_{l^{2}\left(L^{2}\right)}$ shows very similar accuracy for large and moderate numbers of $k$. But for small time steps, large numbers of $N$ leads to slightly worse results. This effect is not visible for $\left\|\boldsymbol{U}-\tilde{\boldsymbol{U}}^{k}\right\|_{l^{2}\left(H^{1}\right)}$, which leads to stagnation for all $N$, even for the original scheme $N=1$. The reason is the limitation of accuracy due to spatial discretization. Here, smaller spatial mesh sizes would be necessary to decrease the error much below $5 \cdot 10^{-3}$. The same effect can be observed for the error in the corrector $\left\|\boldsymbol{U}-\boldsymbol{U}^{k}\right\|_{l^{2}\left(L^{2}\right)}$ : Here, a large number $N$ leads to larger values of minimal errors. In contrast to this, the pressure errors $\left\|P-P^{k}\right\|_{l^{2}\left(H^{1}\right)}$ are independent of $N$. Hence, the local projection scheme leads to the same pressure errors in $l^{2}\left(H^{1}\right)$. Note that the observed stagnation for $k \rightarrow 0$ of all projection schemes is once more due to limitation of spatial accuracy. In Figures 6.5 and 6.6 we present $\operatorname{div} \widetilde{\boldsymbol{u}}(T)$ and $\left(p-p_{h}\right)(T)$ for both original scheme and local projection scheme with $N=16$. Moreover, further results with $H=4 h$ are shown in Fig. 6.8 and no change regarding the accuracy of the scheme was observed.
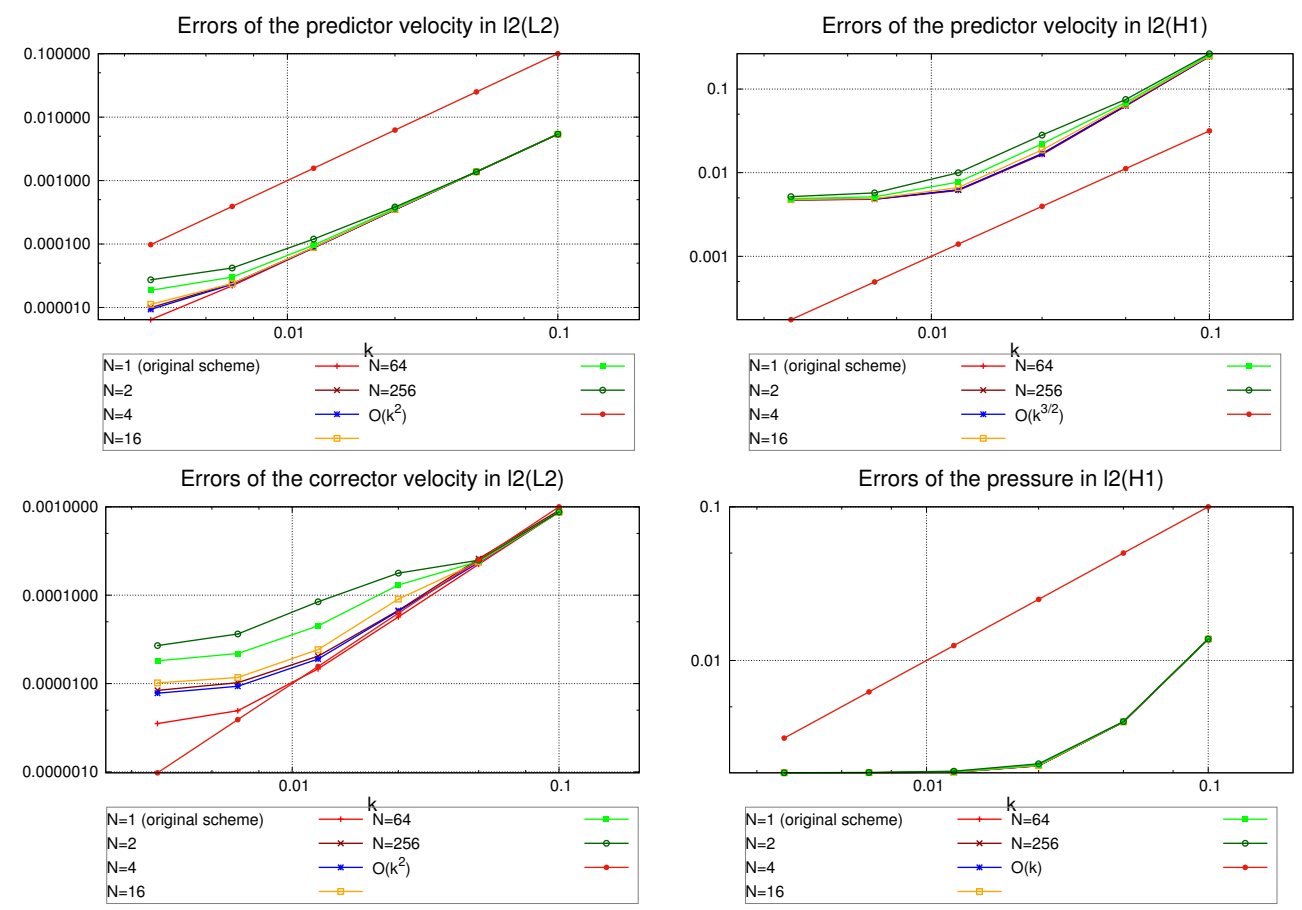

Fig. 6.8. Numerical results for $\nu=10^{-6}, H=4 h$. 


\section{Conclusion and Outlook}

We presented a new pressure correction scheme based on local projections of the velocity to divergence-free velocities. The resulting Poisson problem can be solved completely in parallel. We proved that for velocity the discretization error of the method has the same asymptotic behaviour as the original pressure correction scheme. Numerical results support this findings and give qualitativ information of the error. One of the next steps will be to solve the subproblems in parallel and to document on the speed up of the new scheme.

\section{References}

[1] J.H. Bramble, J.E. Pasciak, O. Steinbach, On the stability of the $L^{2}$ projection in $H^{1}(\Omega)$, Math. Comp., 71:237 (2001), 147-156.

[2] A.J. Chorin, Numerical solution of the Navier-Stokes equations, Math. Comp. 22:104 (1968), $745-762$

[3] J.L. Guermond, P.D. Minev, J. Shen, An overview of projection methods for incompressible flows, CMAME, 195:44 (2006), 6011-6045.

[4] J.L. Guermond, J. Shen, On the error estimates for the rotational pressure correction methods, Math. Comp., 73:248 (2003), 1719-1737.

[5] J.L. Guermond, J. Shen, Velocity-correction projection methods for incompressible flows, SIAM J. Numer. Anal., 41:1 (2003), 112-134.

[6] A. Kashefi, A.E. Staples, A finite-element coarse-grid projection method for incompressible flow simulations, Advances Comp. Math., 44:4 (2018), 1063-1090.

[7] M. Lentine, W. Zheng, R. Fedkiw, A novel algorithm for incompressible flow using only a coarse grid projection, ACM Transactions on Graphics (TOG), 29:4 (2010), 114:1-114:9.

[8] R. Temam, Sur l'approximation de la solution des équations de Navier-Stokes par la méthode des pas fractionnaires (II), Arch. Ration. Mech. Anal. 33:5 (1969), 377-385.

[9] L.J.P. Timmermans, P.D. Minev, F.N. Van deVosse, An approximate projection scheme for incompressible flow using spectral elements, Int. J. Numer. Meth. Fluids, 22 (1996), 673-688. 